\title{
NAVIGATING THE EVOLVING WORLD OF DRUG DISCOVERY
}

\author{
The successful translation of basic scientific discoveries into novel therapeutic \\ strategies for the prevention and treatment of disease depends on integrating all \\ aspects of the drug discovery and development process.
}

Discovering drugs is a complex business. An obvious statement, but worth dwelling on nevertheless. To bring a new drug to market requires many years of coordinated research across a staggering array of disciplines. Chemists and biologists, pharmacologists and toxicologists, clinicians and regulators, all have to get their contribution exactly right, and even then a good deal of luck is needed. To borrow - and bend - a phrase from one of the few branches of science not involved in some way with drug discovery: this is rocket science.

\section{Addressing the problems}

So, no wonder that progress has been slow. Despite all the excitement that accompanies each wave of technical innovation, from protein structure determination to proteomics, and from combinatorial chemistry to e-clinical trials, the fundamental truth is that pharmaceutical companies are not producing drugs any faster than they were before these innovations came along. Even with vastly increased $R \& D$ spending, the top 20 pharmaceutical companies still churn out only around 20 drugs per year between them, far short of the 4-5 new drugs that analysts say they each need to produce to justify their discovery and development costs. So, were expectations too high, or is there something fundamentally wrong? To stretch the analogy with rocket science, who would have thought that 30 years after putting someone on the moon, we'd have such trouble landing unmanned probes on Mars?

The question of where we've gone awry was a "The greatest barrier to success is a lack of communication" remember that most new 'targets' still lack both a known function and any specific chemical modulators, and that drugs act on targets within a biological context. The importance of the latter point is emphasized by the off-target effects that have led to the vastly increased number of costly late-stage failures seen in recent years (approximately half the withdrawals in the past 20 years have occurred since 1997; source, Wood Mackenzie). A key to future success will be the ability to look at drug discovery in an integrated way, always remembering that drugs need to work in whole, living systems. The corollary of this is that members of drug discovery teams also cannot work in isolation. But in order to be able to talk to each other and understand each other's contributions, they need to speak a common language.

\section{Nature Reviews Drug Discovery}

And that's where Nature Reviews Drug Discovery comes in. The sixth and latest member of the Nature Reviews series, the journal is founded on the idea that drug discovery is a uniquely multidisciplinary process in which the greatest barrier to success is a lack of communication between the different areas of specialization. Our main aim is to erode this barrier and so encourage cross-fertilization between disciplines. At the same time, we hope to give a balanced view of the possibilities and pitfalls facing all those involved in discovering and developing drugs. Using the innovative approaches already developed by our fellow Nature Reviews titles, our articles present authoritative updates on key developments in an accessible way, with the presentation of ideas tailored to suit our particularly broad audience. We believe it to be self-evident that the successful drug discoverers of the future will need to be very well read — and we aim to provide your guide to the evolving world of drug discovery. 